\title{
Carotid intima-media thickness and hemodynamic parameters: reproducibility of manual measurements with Doppler ultrasound
}

\author{
Juana M Plasencia Martínez, José M García Santos, Maria L Paredes Martínez, \\ Ana Moreno Pastor
}

Radiology Department. University General Hospital Morales Meseguer. Murcia, Spain

\begin{abstract}
Aims: To evaluate the carotid ultrasound intra- and interobserver agreements in a common clinical scenario when making manual measurements of the intima-media thickness (IMT) and peak systolic (PSV) and end diastolic (EDV) velocities in the common (CCA) and the internal carotid (ICA) arteries. Material and methods: Three different experienced operators performed two time-point carotid ultrasounds in 21 patients with cardiovascular risk factors. Each operator measured freehand the CCA IMT three consecutive times in each examination. The CCA and ICA hemodynamic parameters were acquired just once. For our purpose we took the average (IMTmean) and maximum (IMTmax) IMT values. Quantitative variables were analyzed with the $t$-student, and ANOVA test. Agreements were evaluated with the Intraclass Correlation Coefficient (ICC). Results: IMTmean intraobserver agreement was better on the left (ICC: $0.930-0.851-0.916$, operators 1-2-3) than on the right (ICC: 0.789-0.580-0.673, operators 1-2-3). IMTmax agreements (Left ICC: 0.821-0.723-0.853, operators 1-2-3; Right ICC: 0.6690.421-0.480, operators 1-2-3) were lower and more variable. Interobserver agreements for IMTmean (ICC: $0.852-0.860$; first-second ultrasound) and IMTmax (ICC: 0.859-0.835; first-second ultrasound) were excellent on the left, but fair-good and more variable on the right (IMTmean; ICC: 0.680-0.809; first-second ultrasound; IMTmax; 0.694-0.799; first-second ultrasound). Intraobserver agreements were fair-moderate for PSVs and good-excellent for EDVs. Interobserver agreements were good-excellent for both PSVs and EDVs. Overall, 95\% confidence intervals were narrower for the left IMTmean and CCA velocities. Conclusions: Intra and interobserver agreements in carotid ultrasound are variable. In order to improve carotid IMT agreements, IMTmean is preferable over IMTmax.
\end{abstract}

Keywords: ultrasonography, Doppler, carotid intima-media thickness, observer variation, reproducibility

\section{Introduction}

Carotid atherosclerosis is considered to be representative for systemic atherosclerosis [1,2]. Due to the carotid accessibility, ultrasonography (US) examination is a safe technique, being recommended by the American Society of Echocardiography (ASE) to determine the vascular age and the risk for cardiovascular events [3-

Received 26.01.2015 Accepted 10.04.2015

Med Ultrason

2015, Vol. 17, No 2, 167-174

Corresponding author: Juana M Plasencia Martínez

Servicio de Radiodiagnóstico. Hospital General

Universitario Morales Meseguer.

C/ Marqués de los Vélez s/n. 30008

Murcia. España

Phone: +34620402970

Fax number: +34968239975

E-mail: plasen79@gmail.com
6]. Together with other imaging techniques, the results offered by US are useful for setting primary prevention therapy [7]. US could estimate the cardiovascular risk by detecting atheroma plaques and measuring intima-media thickness (IMT) at the level of the common carotid artery (CCA) [7-9]. On the other hand, parameters such as peak systolic (PSV) and end diastolic (EDV) velocities assess the hemodynamic effects of stenosis and are relevant for management decisions [10]. However, carotid US is often performed by sonographers, in and out of Radiology departments, with different levels of training and experience and different approaches (manual, semiautomated or automated), whose reliability is many times simply assumed. But a manual subjective measurement, which is the most common approach in a clinical scenario, might be associated with significant variability. The reported variability of IMT measurements has been different among studies $[4,6,11,12-14]$ and, even more 
relevant, the statistical assessment has been also quite diverse, most frequently focused on correlations than on agreements [15]. That has also been the case of hemodynamic parameters $[16,17]$. Therefore, reproducibility studies are still needed, especially if carotid US is going to be used as a biomarker of cardiovascular risk. Accordingly, our purpose was to evaluate intra and interobserver agreements in carotid US, reproducing a usual clinical scenario. We hypothesize that IMT and hemodynamic parameters reproducibility may be not negligible.

\section{Material and methods}

\section{Patients}

We wanted to build a sample made up of patients with expected carotid atherosclerosis. These subjects would account for the common clinical scenario in which manual measurements are performed and cardiovascular risk estimated. Their carotid walls are usually irregular and a strong subjective visual component needs to be considered when manual measurements are attempted. For that purpose, we took advantage of subjects of a cardiology sample in our Institution who had undertaken computer tomography (CT) for calcium scoring and coronary artery angio-CT during the last 11 months. CT had been performed in atypical chest pain and when electrocardiogram, echocardiography, or stress tests were uncertain, non-interpretable, impossible to be performed, or discordant between them or with laboratory testing. Except for one patient with right coronary artery chronic occlusion, the remaining subjects had no known coronary disease. Carotid stenting or surgical interventions were exclusion criteria, but, finally, no patient was excluded. The final sample was made up of 21 patients (14 male; mean age $61.2 \pm 9.4$ years, range $42-77$ years, women $44-77$ years, men 42 - 76 years). The study was approved by the Institutional Ethics Committee. Before US, all subjects were interviewed and informed on the characteristics and objective of the study and signed the written informed consent. Each patient received a report of his/her first US examination in the second appointment.

\section{Doppler US}

All exams were performed with the same ultrasound scanner and probe, Philips IU22, L 9-3 MHz (Philips Healthcare, Eindhoven, The Netherlands), between February and June, 2012. In order to reproduce a common real technical scenario, we first wanted to introduce different levels of experience between operators. Therefore, each patient was individually and independently explored by three operators $(\mathrm{Op})$ with 7, 2, and 1 years experience in general and Doppler US; the first one (Op1) was a certified radiologist while the other two were, respectively, an US trained radiology resident (Op2) and a radiology resident still under US training (Op3). Each operator carried out two exams in each patient, two different days apart, not before 30 days from the first exam, and not beyond 10 weeks between both exams. They were always blinded to the other operators' results, and, when performing the second examination, also to their previous results. Performing US, all operators followed the recommendations of the ASE [9]. Accordingly, the patient stayed in supine position, the neck slightly hyperextended and rotated to the opposite side. Also looking for that common technical scenario, the operators only agreed on what the IMT limits were and how to obtain the IMT and hemodynamic measurements. Otherwise, they were free to sample Doppler signal with the incidence angles they considered more appropriate, provided it was $\leq 60^{\circ}$ and not beyond $1 \mathrm{~cm}$ from the carotid bifurcation, and to assess IMT whatever time of the cardiac cycle and with the zoom level they felt more comfortable. All data and zoom levels were recorded in our local Picture Archiving and Communication System (PACS) and archived in a Microsoft Office Excel database (Windows 2010. Microsoft Office Corporation. Redmond, Washington, EEUU).

IMT was considered as the maximum distance between the intima and adventitia internal edges, at the distal centimeter of the posterior CCA wall. When detected, the operator avoided the atheroma plaques for IMT measurement, but not for the hemodynamic assessment. Each operator successively froze three CCA longitudinal images, identified the thickest wall, and measured the IMT freehand in each image. Along that process, the operators were instructed to avoid looking at the quantitative values in the screen. The averaged thickness (IMT-

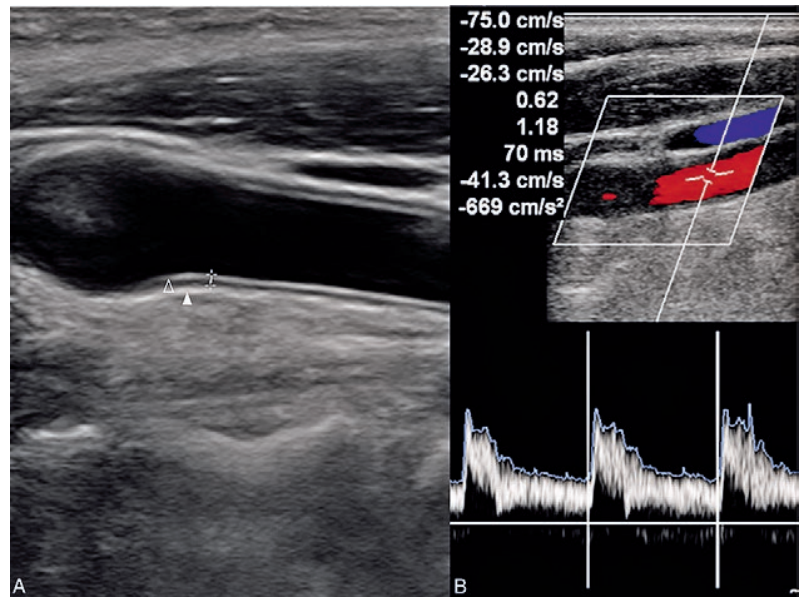

Fig 1. B-mode (A) and Doppler (B) ultrasound in the left distal common carotid artery. Intima-media thickness. Both intimamedia (empty arrowhead) and media-adventitia (arrowhead) show up as echogenic interfaces at ultrasound. 
mean) and the thickest width (IMTmax) were used for the analysis. Later on, they got the PSV and EDV in the distal CCA and proximal ICA on both sides, as close as possible to the carotid bifurcation, always keeping an angle $\leq 60^{\circ}$, and using the spectrum with the best spectral window (fig 1). The carotid bulb was avoided.

\section{Statistical analysis}

Variables in the Excel tables were exported to SPSS version 17.0 for Windows (IBM, Armonk, NY). They were presented as mean \pm standard deviation. Normal distribution was checked by the Kolmogorov-Smirnov test. The two-tailed Student $t$ test was used for any right and left comparisons while differences in IMT and hemodynamic parameters between the three operators were analyzed with the ANOVA test and Bonferroni correction for multiple comparisons. Finally, we assessed the intra and interobserver agreement for IMT and hemodynamic parameters. All agreement analysis was made with the Intraclass Correlation Coefficient (ICC) with 95\% confidence intervals $(95 \% \mathrm{CI})$. Agreement levels were classified as poor $(<0.20)$, fair $(0.21-0.40)$, moderate $(0.41$ $0.60)$, good (0.61- 0.80) and excellent $(>0.80)$. Statistical significance was established for a $\mathrm{p}$ value $<0.05$.

\section{Results}

Demographic data are shown in table I.

a. Right to left differences

a.1 Intima-media thickness. The averaged IMTmean and IMTmax were significantly thicker on the left (IMTmean $0.9271 \pm 0.2593$ vs $0.8404 \pm 0.1736, P<0.001$; IMT$\max 1.0050 \pm 0.2910$ vs $0.9195 \pm 0.1895, P<0.015)$. On an operator basis, IMTmean and IMTmax were also thicker on the left, but differences were only significant in the second examination of operators 1 and $2(P=0.025$ and $P=0.031$ for IMTmean; $P=0.041$ and $P=0.016$ for IMTmax).

a. 2 Velocities. PSV and EDV did not show right to left differences, excluding a higher PSV in the right CCA

Table I. Demographic characteristics of the sample (21 patients).

\begin{tabular}{lll}
\hline & & $\mathrm{N}(\%)$ \\
\hline Previous coronary event & yes & $3(14.3)$ \\
Gender & male & $14(66.7)$ \\
& female & $7(33.3)$ \\
Smoker & yes & $5(23.8)$ \\
Diabetes & yes & $5(23.8)$ \\
Total hypercholesterolemia & yes & $15(71.4)$ \\
HDL-Cholesterol & high & $2(11.1)$ \\
& low & $2(11.1)$ \\
Arterial hypertension & loss & $3(14.3)$ \\
\hline
\end{tabular}

$\mathrm{N}$ - number of patients

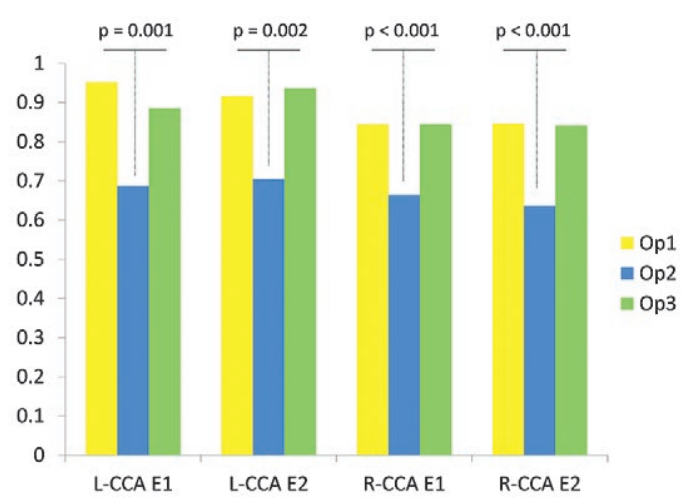

Fig 2. Mean intima-media thickness (IMTmean) measurements. Differences between operators (Op). In all cases, the IMTmean and maximum IMT (not shown) for the second operator were significantly lower. E1: first examination; E2: second examination.

(operator 3; second examination), and a higher EDV in the left ICA, (operators 2 and 3 ; first examination).

b. Differences between operators (table II)

b.1 Intima-media thickness. All IMT magnitudes of operator 2 were different from the other two sonographers measurements (fig 2). Operator 2 always used zoomed images while operators 1 and 3 zoomed images in $38 \%$ and $31 \%$ of cases, respectively. The operator 2 zoom level was also higher $(\geq 1.7 x$ in $69 \%$ of cases vs. $11 \%$ and $2 \%$ in operators 1 and 3 , respectively).

b. 2 Velocities. Only right ICA EDV in the second examination was significantly different between the three operators, but that difference lost significance when multiple comparison analysis was performed.

c. Agreement analysis (tables III and IV; $95 \% \mathrm{CI}$ are only provided in the tables).

c.1 Intima-media thickness. The intraobserver IMTmean agreement was good to excellent on the left (Op1, 0.930; Op2, 0.851; Op3 0.916), and moderate (Op2, $0.580)$ to good (Op1, 0.789; Op3, 0.673) on the right. The IMTmax agreement was lower (Left: Op1, 0.821; Op2, 0.723; Op3, 0.853. Right: Op1, 0.669; Op2, 0.421; Op3, 0.480). 95\%CI were only narrow for IMTmean, especially on the left and for operators 1 and 3. Operator 2 nearly always obtained the lowest agreement and the widest confidence intervals (table III).

The interobserver agreement for IMTmean and IMTmax was excellent on the left in both examinations (First IMTmean: 0.852; first IMTmax: 0.859. Second IMTmean: 0.860; second IMTmax: 0.835), and worse on the right (First IMTmean: 0.680; second IMTmean: 0.809. First IMTmax: 0.694; second IMTmax: 0.799). 95\% CI intervals were also wider on the right (table IV). 


\begin{tabular}{|c|c|c|c|c|c|c|}
\hline & & & Operator 1 & Operator 2 & Operator 3 & $\mathrm{p}^{*}$ \\
\hline \multirow[t]{4}{*}{ IMTmean } & \multirow[t]{2}{*}{ L-CCA } & US 1 & $0.952(0.269)$ & $0.688(0.136)$ & $0.886(0.228)$ & 0.001 \\
\hline & & US 2 & $0.917(0.198)$ & $0.704(0.145)$ & $0.937(0.286)$ & 0.002 \\
\hline & \multirow[t]{2}{*}{$\mathrm{R}-\mathrm{CCA}$} & US 1 & $0.844(0.148)$ & $0.664(0.136)$ & $0.844(0.150)$ & $<0.001$ \\
\hline & & US 2 & $0.847(0.155)$ & $0.637(0.129)$ & $0.842(0.163)$ & $<0.001$ \\
\hline \multirow[t]{4}{*}{ IMTmax } & \multirow[t]{2}{*}{ L-CCA } & US 1 & $1.024(0.276)$ & $0.766(0.148)$ & $0.964(0.247)$ & 0.002 \\
\hline & & US 2 & $1.020(0.233)$ & $0.773(0.165)$ & $1.020(0.305)$ & 0.002 \\
\hline & \multirow[t]{2}{*}{$\mathrm{R}-\mathrm{CCA}$} & US 1 & $0.933(0.168)$ & $0.736(0.157)$ & $0.921(0.163)$ & $<0.001$ \\
\hline & & US 2 & $0.926(0.163)$ & $0.686(0.137)$ & $0.928(0.204)$ & $<0.001$ \\
\hline \multirow[t]{8}{*}{ PSV } & \multirow[t]{2}{*}{ L-CCA } & US 1 & $103.476(18.057)$ & $99.491(23.140)$ & $103.714(27.103)$ & NS \\
\hline & & US 2 & $105.550(17.548)$ & $100.775(16.956)$ & $97.890(17.360)$ & NS \\
\hline & \multirow[t]{2}{*}{ R-CCA } & US 1 & $109.571(23.750)$ & $96.291(22.510)$ & $103.581(26.935)$ & NS \\
\hline & & US 2 & $99.000(16.673)$ & $103.853(24.861)$ & $106.390(24.747)$ & NS \\
\hline & \multirow[t]{2}{*}{ L-ICA } & US 1 & $93.857(27.623)$ & $93.081(28.173)$ & $91.467(26.899)$ & NS \\
\hline & & US 2 & $95.000(23.149)$ & $91.605(25.513)$ & $90.130(19.585)$ & NS \\
\hline & \multirow[t]{2}{*}{ R-ICA } & US 1 & $90.381(26.080)$ & 94.267 (34.978) & $91.576(25.303)$ & NS \\
\hline & & US 2 & 90.737 (19.686) & $93.330(24.746)$ & $89.250(23.440)$ & NS \\
\hline \multirow[t]{8}{*}{ EDV } & \multirow[t]{2}{*}{ L-CCA } & US 1 & 27.095 (8.264) & $26.395(9.962)$ & $27.000(12.681)$ & NS \\
\hline & & US 2 & $26.850(6.393)$ & $24.885(6.523)$ & $23.800(6.711)$ & NS \\
\hline & \multirow[t]{2}{*}{$\mathrm{R}-\mathrm{CCA}$} & US 1 & $29.476(10.796)$ & $26.955(9.251)$ & $26.274(8.134)$ & NS \\
\hline & & US 2 & $27.263(5.772)$ & $25.100(7.713)$ & $26.540(6.796)$ & NS \\
\hline & \multirow[t]{2}{*}{ L-CCA } & US 1 & $28.143(9.345)$ & $25.491(7.044)$ & $24.045(10.352)$ & NS \\
\hline & & US 2 & $28.600(9.116)$ & $25.380(9.009)$ & $28.280(10.997)$ & NS \\
\hline & \multirow[t]{2}{*}{ R-ICA } & US 1 & $26.095(8.166)$ & $21.814(7.122)$ & $27.786(8.637)$ & NS \\
\hline & & US 2 & $29.158(9.179)$ & $22.840(6.247)$ & $27.380(8.553)$ & $0.04 \dagger$ \\
\hline
\end{tabular}

Data are shown as mean $(\mathrm{mm}) \pm$ standard deviation (between brackets).

IMTmean: averaged intima-media thickness; IMTmax: maximum intima-media thickness; PSV: Peak systolic velocity; EDV: End diastolic velocity; CCA: Common carotid artery; ICA: Internal carotid artery; R: Right; L: Left; US1: first examination; US2: second examination; NS: non-significant.

* Bonferroni test: differences significant for operator 2.

$\dagger$ Difference was non-significant with Bonferroni test.

c. 2 Velocities. The intraobserver trends of operators 1 and 3 were similar and differed from operator 2 . For both operators, PSV agreement in the CCA was fair on the left $(\mathrm{Op} 1,0.305 ; \mathrm{Op} 3,0.376)$ and moderate on the right (Op1, 0.499; Op3 0.485). PSV agreement was moderate in both ICA (Left: Op1, 0.455; Op3, 0.552. Right: Op1, 0.493; Op3, 0.546). Agreement improved with EDV, being moderate to good in the CCA (Left: Op1, 0.683; Op3, 0.607. Right: Op1, 0.596; Op3, 0.731) and good to excellent in the ICA (Left: Op1, 0.803; Op3, 0.671. Right: Op1, 0.740; Op3, 0.812). Operator 2 agreements were more anarchic, tending to be better with EDV, but with wide $95 \%$ CI in all cases (fig. 3 , table III).

Interobserver agreements with the PSV (Left, first exam: 0.885; second exam: 0.864. Right, first exam: 0.936; second exam 0.943) and EDV (Left, first exam: 0.893; second exam: 0.887. Right, first exam: 0.906; second exam 0.872 ) were excellent in the CCA, and good to excellent in the ICA (Left, first exam: 0.854; second exam: 0.796. Right, first exam: 0.842; second exam: 0.840). 95\% CI were narrow in the CCA and wider in the ICAs (fig 4, table IV).

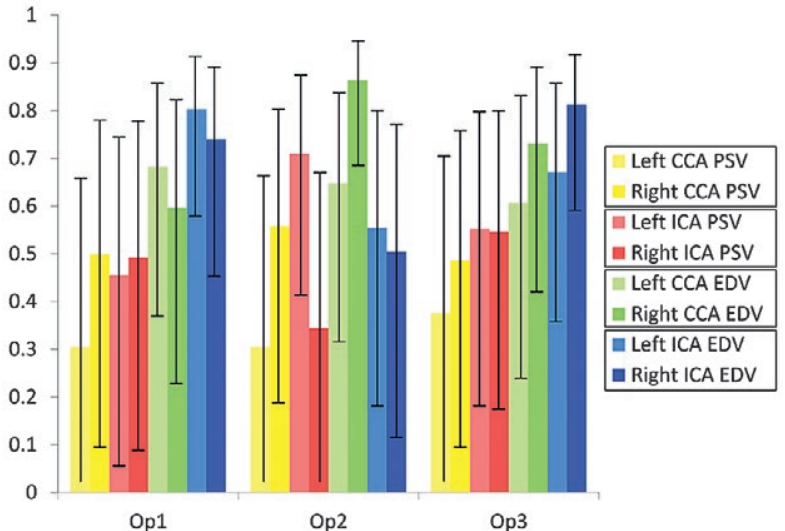

Fig 3. Intraobserver agreements for peak systolic (PSV) and end diastolic velocities (EDV) in common (CCA) and internal carotid (ICA) arteries according to the intraclass correlation coefficient (ICC). Comparison between the first and the second examination of each operator. Agreement progressively increases from the left to the right CCA, and from PSV to EDV in operators (Op) 1 and 3. 95\% confidence intervals (CI): black segments superimposed on the bars. Quantitative values of ICC and $95 \% \mathrm{CI}$ are included in the manuscript and in table III. 
Med Ultrason 2015; 17(2): 167-174

\begin{tabular}{lllll}
\hline Table III. Intraobserver agreement & & & \\
\hline & & Operator 1 & Operator 2 & Operator 3 \\
\hline L-CCA & IMTmean & $0.930(0.822-0.972)$ & $0.851(0.622-0.941)$ & $0.916(0.789-0.967)$ \\
& IMTmax & $0.821(0.603-0.925)$ & $0.723(0.423-0.881)$ & $0.853(0.666-0.939)$ \\
R-CCA & IMTmean & $0.789(0.452-0.919)$ & $0.580(0.060-0.834)$ & $0.673(0.174-0.871)$ \\
& IMTmax & $0.669(0.320-0.858)$ & $0.421(0.140-0.722)$ & $0.480(0.060-0.756)$ \\
L-CCA & PSV & $0.305(-0.160-0.660)$ & $0.305(-0.160-0.660)$ & $0.376(-0.067-0.696)$ \\
& EDV & $0.683(0.356-0.861)$ & $0.648(0.299-0.844)$ & $0.607(0.223-0.828)$ \\
R-CCA & PSV & $0.499(0.070-0.772)$ & $0.558(0.165-0.798)$ & $0.485(0.066-0.758)$ \\
& EDV & $0.596(0.206-0.822)$ & $0.863(0.679-0.945)$ & $0.731(0.413-0.890)$ \\
L-ICA & PSV & $0.455(0.028-0.742)$ & $0.710(0.401-0.874)$ & $0.552(0.156-0.795)$ \\
& EDV & $0.803(0.567-0.917)$ & $0.554(0.160-0.796)$ & $0.671(0.335-0.855)$ \\
R-ICA & PSV & $0.493(0.063-0.769)$ & $0.344(-0.092-0.670)$ & $0.546(0.148-0.792)$ \\
& EDV & $0.740(0.441-0.891)$ & $0.504(0.091-0.769)$ & $0.812(0.584-0.921)$ \\
\hline
\end{tabular}

Data correspond to intraclass correlation coefficient and 95\% confidence interval (between brackets).

IMTmean: averaged intima-media thickness; IMTmax: maximum intima-media thickness; PSV: Peak systolic velocity; EDV: End diastolic velocity; CCA: Common carotid artery; ICA: Internal carotid artery; R: Right; L: Left.

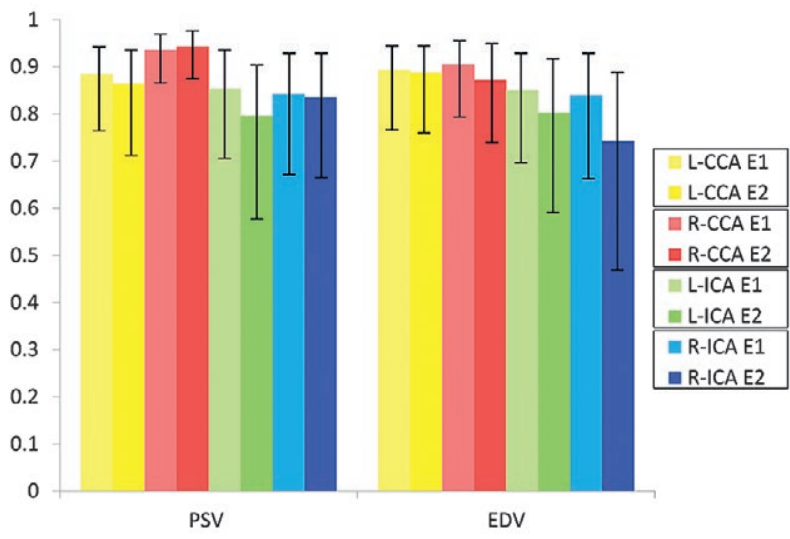

Fig 4. Interobserver agreement for peak systolic (PSV) and end diastolic velocities (EDV) in common (CCA) and internal carotid (ICA) arteries according to the intraclass correlation coefficient. Agreements are generally excellent but decrease from the CCA to the ICA, and from the right $(\mathrm{R})$ to the left $(\mathrm{L})$, both for the PSV and the EDV. Confidence intervals (black segments superimposed on bars) increase at the same time. Quantitative values of the intraclass correlation coefficient and the 95\% CI are included in the manuscript and in table IV. E1: first examination; E2: second examination.

\section{Discussions}

According to our results, the intra and interobserver agreements with IMT (especially IMTmean on the left) and hemodynamic parameters (especially in the CCA) went from good to excellent, but they were variations between arteries and operators, generally with wide confidence intervals.

Our main purpose was to assess the reproducibility of manual carotid US measurements in patients with cardio-
Table IV. Interobserver agreement

\begin{tabular}{|c|c|c|c|c|}
\hline & & & CCI & $95 \%$ CI \\
\hline \multirow[t]{4}{*}{ L-CCA } & IMTmean & US1 & 0.852 & $0.694-0.935$ \\
\hline & & US2 & 0.860 & $0.705-0.940$ \\
\hline & IMTmax & US1 & 0.859 & $0.709-0.939$ \\
\hline & & US2 & 0.835 & $0.653-0.930$ \\
\hline \multirow[t]{4}{*}{ R-CCA } & GIM med & US1 & 0.680 & $0.338-0.860$ \\
\hline & & US2 & 0.809 & $0.590-0.921$ \\
\hline & IMTmax & US1 & 0.694 & $0.367-0.866$ \\
\hline & & US2 & 0.799 & $0.568-0.916$ \\
\hline \multirow[t]{4}{*}{ L-CCA } & PSV & US1 & 0.885 & $0.762-0.950$ \\
\hline & & US2 & 0.864 & $0.713-0.942$ \\
\hline & EDV & US1 & 0.893 & $0.774-0.954$ \\
\hline & & US2 & 0.887 & $0.761-0.952$ \\
\hline \multirow[t]{4}{*}{ R-CCA } & PSV & US1 & 0.936 & $0.868-0.972$ \\
\hline & & US2 & 0.943 & $0.878-0.976$ \\
\hline & EDV & US1 & 0.906 & $0.793-0.962$ \\
\hline & & US2 & 0.872 & $0.725-0.947$ \\
\hline \multirow[t]{4}{*}{ L-ICA } & PSV & US1 & 0.854 & $0.699-0.936$ \\
\hline & & US2 & 0.796 & $0.570-0.913$ \\
\hline & EDV & US1 & 0.850 & $0.689-0.934$ \\
\hline & & US2 & 0.803 & $0.584-0.916$ \\
\hline \multirow[t]{4}{*}{ R-ICA } & PSV & US1 & 0.842 & $0.673-0.931$ \\
\hline & & US2 & 0.840 & $0.657-0.934$ \\
\hline & EDV & US1 & 0.835 & $0.658-0.928$ \\
\hline & & US2 & 0.742 & $0.447-0.893$ \\
\hline
\end{tabular}

Agreements correspond to intraclass correlation coefficient (ICC) with $95 \%$ confidence interval (CI).

IMTmean: averaged intima-media thickness; IMTmax: maximum intima-media thickness; PSV: Peak systolic velocity; EDV: End diastolic velocity; CCA: Common carotid artery; ICA: Internal carotid artery; R: Right; L: Left; US1: first examination; US2: second examination. 
vascular risk factors. In a previous study whose sample was classified in cardiovascular risk quartiles according to IMT, minimal differences in magnitude moved patients to distant quartiles. In that paper, being included in the highest or the lowest quartiles depended on IMT differences of just $0.3 \mathrm{~mm}$ [18]. Therefore, variability of carotid ultrasound IMT magnitudes might make using it as a biomarker of cardiovascular risk questionable. Moreover, a recent large meta-analysis has questioned the prognostic utility of IMT as a risk biomarker and has argued that the extent of carotid plaque, when compared to IMT, showed a greater accuracy for the prediction of future coronary ischemic events [19]. In this scenario, the analysis of carotid US agreements becomes relevant.

A number of previous papers have analyzed the reproducibility of manual and automatized carotid IMT measurements $[5,11,12,14,20,21]$. However, only a few made just a correlation analysis and agreement was not actually assessed $[11,12,20]$. For other researchers, correlation based on coefficients of variation or difference of means were acceptable [14,21] or very high [5], but the interobserver agreement was not studied in the latter. Unlike those studies, we assessed intra and interobserver agreements with the intraclass correlation coefficient and 95\% CI, and agreement, although overall acceptable, was variable. IMTmean was always more reproducible than IMTmax, probably because it is just one measurement, it may be more variable than IMTmean [9]. IMTmax is the normal approach in our department and has also been the reference parameter in some studies [20]. However, IMTmean (averaged three IMT measurements in the distal CCA centimeter) is recommended by the ASE for IMT manual quantification [9] and, as our results corroborate, less variable $[15,21]$.

IMT agreement in this study was always better on the left. Right-to-left thickness differences might be the first reason for agreement inconsistencies [5]. Though not always the case [22], some reports have suggested that atheromatosis might be more severe on the left CCA [23-25]. Moreover, a stiffer and less elastic wall would make it less variable during the cardiac cycle. In fact, the effect of cardiac cycle on an IMT agreement can be another possible source of variation [25]. Accordingly, a thicker and less changing left carotid wall might allow the operator to be more accurate when placing the calipers at the IMT edges. Further supporting that hypothesis, when reproducibility has been reported equal in both sides, the consistence of measurements was higher on the side where the carotid wall was thicker [5]. And levels of agreement were better bilaterally when IMT was also bilaterally increased [11]. However, other authors have reported a negative relation between agreement and thickness $[14,26]$ and taking into account that the higher agreement on the left has been also reported with semiautomated software [27], other factors than thickness could influence agreement and laterality. Accordingly, the operator's position on the left may change the angle of insonation or the applied pressure [28,29]. And zooming or experience might also have to be considered in the background of inconsistencies. In our case, the operator systematically enlarging the image also showed different degrees of agreement and lower thickness values, which is consistent with other reports [9]. On the other hand, our results do not suggest an important impact regarding the operator's experience. Specific and fast training protocols may improve IMT agreement even with inexperienced operators [30] suggesting that highly experienced sonographers might not significantly differ from less trained operators. However, more dedicated designs are required to achieve more reliable conclusions on zooming or experience, even more considering that experience has been previously related with disagreements [29,31].

Regarding the hemodynamic parameters, our intra and interobserver agreements were slightly and clearly worse, respectively, than others previously reported [32], always with wide confidence intervals. Intraobserver concordance was higher with EDV than PSV, perhaps related to the narrower range of EDV magnitudes. However, we cannot easily explain why the interobserver was better than the intraobserver agreement. Maybe, it was partially an effect of the small sample, but, once again, we cannot rule out the impact of those previously commented factors, related to technical, anatomical, physiological and pathological reasons, which, for the hemodynamic parameters, could be even more relevant.

Our study has several limitations. First, though we wanted to involve more patients, their special characteristics and the number of examinations in different days made it difficult to get a larger sample. As a result, the small size lowers our statistical power. However, reproducibility studies may be attempted with at least 20 subjects $[33,34]$ and, with that sample size, we can assume a $20 \%$ error margin [35], which is acceptable for the preliminary objective of this work. Moreover, a small sample avoids the learning effect of successive carotid explorations identified in other studies [29]. Furthermore, our effective sample size for a complete two time-point analysis was not significantly different to other two time-point agreement analysis in which the elderly with expected cardiovascular disease were included $[14,36]$. Second, the technical simplicity and the operator's autonomy could have introduced unpredictable sources of variability. The foreseeable impact of subjectivity in manual quantification makes reasonable thinking that automated techniques 
improve accuracy of measurements [9,19,37,38]. Several studies have shown that automated software improves reproducibility $[4,5,9]$. However, not all studies agree [39] and, on the other hand, automated measurements deviations would have to be manually corrected, involving variability once again. In addition, our purpose was to reproduce a common technical scenario in clinical practice and, in our opinion, more than a real limitation, our manual approach adds a practical value to our results.

In conclusion, when carotid ultrasound is performed in patients with cardiovascular risk factors, intra and interobserver agreements are variable. In order to improve carotid IMT agreements, IMTmean is preferable over IMT max, but, even in that case, variability is not negligible, probably depending on technical and operator factors that have to be addressed in further investigations.

\section{Conflict of interest: none}

\section{Acknowledgments}

We are indebted to Andrés Carrillo MD, for his valuable help with the statistical analysis.

\section{References}

1. Nagai Y, Kitagawa K, Sakaguchi M, et al. Significance of earlier carotid atherosclerosis for stroke subtypes. Stroke 2001; 32: 1780-1785.

2. Wofford JL, Kahl FR, Howard GR, McKinney WM, Toole JF, Crouse JR 3rd. Relation of extent of extracranial carotid artery atherosclerosis as measured by B-mode ultrasound to the extent of coronary atherosclerosis. Arterioscler Thromb 1991; 11: 1786-1794.

3. O'Leary DH, Polak JF, Kronmal RA, Manolio TA, Burke GL, Wolfson SK Jr. Carotid-artery intima and media thickness as a risk factor for myocardial infarction and stroke in older adults. Cardiovascular Health Study Collaborative Research Group. N Engl J Med 1999; 340: 14-22.

4. Freire CM, Ribeiro AL, Barbosa FB, et al. Comparison between automated and manual measurements of carotid intima-media thickness in clinical practice. Vasc Health Risk Manag 2009; 5: 811-817.

5. Gonzalez J, Wood JC, Dorey FJ, Wren TA, Gilsanz V. Reproducibility of carotid intima-media thickness measurements in young adults. Radiology 2008; 247: 465-471.

6. Polak JF, Funk LC, O'Leary DH. Inter-reader differences in common carotid artery intima-media thickness: implications for cardiovascular risk assessment and vascular age determination. J Ultrasound Med 2011; 30: 915-920.

7. Naghavi M, Falk E, Hecht HS, et al. From vulnerable plaque to vulnerable patient--Part III: Executive summary of the Screening for Heart Attack Prevention and Educa- tion (SHAPE) Task Force report. Am J Cardiol 2006; 98: $2 \mathrm{H}-15 \mathrm{H}$.

8. Hodis HN, Mack WJ, LaBree L, et al. The role of carotid arterial intima-media thickness in predicting clinical coronary events. Ann Intern Med 1998; 128: 262-269.

9. Stein JH, Korcarz CE, Hurst RT, et al. Use of carotid ultrasound to identify subclinical vascular disease and evaluate cardiovascular disease risk: a consensus statement from the American Society of Echocardiography Carotid IntimaMedia Thickness Task Force. Endorsed by the Society for Vascular Medicine. J Am Soc Echocardiogr 2008; 21: 93111.

10. Carpenter JP, Lexa FJ, Davis JT. Determination of duplex Doppler ultrasound criteria appropriate to the North American Symptomatic Carotid Endarterectomy Trial. Stroke 1996; 27: 695-699.

11. Riley WA, Barnes RW, Applegate WB, et al. Reproducibility of noninvasive ultrasonic measurement of carotid atherosclerosis. The Asymptomatic Carotid Artery Plaque Study. Stroke 1992; 23: 1062-1068.

12. Latich I, Scoutt LM. Ultrasound evaluation of carotid intima media thickness: The Hows and Whys. Ultrasound Clin 2011; 6: 445-461.

13. Thanassoulis G, Peloso GM, Pencina MJ, et al. A genetic risk score is associated with incident cardiovascular disease and coronary artery calcium: the Framingham Heart Study. Circ Cardiovasc Genet 2012; 5: 113-121.

14. Stensland-Bugge E, Bønaa KH, Joakimsen O. Reproducibility of ultrasonographically determined intima-media thickness is dependent on arterial wall thickness. The Tromsø Study. Stroke 1997; 28: 1972-1980.

15. Kanters SD, Algra A, van Leeuwen MS, Banga JD. Reproducibility of in vivo carotid intima-media thickness measurements: a review. Stroke 1997; 28: 665-671.

16. Lee VS, Hertzberg BS, Kliewer MA, Carroll BA. Assessment of stenosis: implications of variability of Doppler measurements in normal-appearing carotid arteries. Radiology 1999; 212: 493-498.

17. Hadlock J, Beach KW; D.E. Strandness Vascular Laboratory Sonographers. Velocity variability in ultrasonic Doppler examinations. Ultrasound Med Biol 2009; 35: 949-954.

18. O'Leary DH, Polak JF, Kronmal RA, et al. Distribution and correlates of sonographically detected carotid artery disease in the Cardiovascular Health Study. The CHS Collaborative Research Group. Stroke 1992; 23: 1752-1760.

19. Inaba Yoichi, Chen JA, Bergmann SR. Carotid plaque, compared with carotid intima-media thickness, more accurately predicts coronary artery disease events: a metaanalysis. Atherosclerosis 2012; 220: 128-133.

20. Velázquez F, Berná JD, Abellán JL, Serrano L, Escribano A, Canteras M. Reproducibility of sonographic measurements of carotid intima-media thickness. Acta Radiol 2008; 49: 1162-1166.

21. Kanters SD, Elgersma OE, Banga JD, van Leeuwen MS, Algra A. Reproducibility of measurements of intimamedia thickness and distensibility in the common carotid artery. Eur J Vasc Endovasc Surg 1998; 16: 28-35. 
22. Ameneiro Perez S, Fernández Bolona A. Comparación de la medición bilateral del grosor íntima-media carotídeo. Rev Cubana Angiol Cir Vasc 2008; 9. Available at: http:// www.bvs.sld.cu/revistas/ang/vol9_1_08/ang0208.htm. Accessed: 2012.09.03.

23. Chaubey S, Nitsch D, Altmann D, Ebrahim S. Differing effect of modifiable cardiovascular risk factors on intimamedia thickening and plaque formation at different sites of the arterial vasculature. Heart 2010; 96: 1579-1585.

24. Lee SW, Hai JJ, Kong SL, et al. Side differences of carotid intima-media thickness in predicting cardiovascular events among patients with coronary artery disease. Angiology 2011; 62: 231-236.

25. Selamet Tierney ES, Gauvreau K, Jaff MR, et al. Carotid artery intima-media thickness measurements in the youth: reproducibility and technical considerations. J Am Soc Echocardiogr 2015; 28: 309-316.

26. Stensland-Bugge E, Bonaa KH, Joakimsen O. Reproducibility of ultrasonographically determined intima-media thickness is dependent on arterial wall thickness. The Tromso Study. Stroke 1997; 28: 1972-1980.

27. Di Geso L, Zardi EM, Afeltra A, et al. Comparison between conventional and automated software-guided ultrasound assessment of bilateral common carotids intima-media thickness in patients with rheumatic diseases. Clin Rheumatol 2012; 31: 881-884.

28. Iglesias del Sol A, Bots ML, Grobbee DE, Hofman A, Witteman JC. Carotid intima-media thickness at different sites: relation to incident myocardial infarction; The Rotterdam Study. Eur Heart J 2002; 23: 934-940.

29. Nichols S, Milner M, Meijer R, Carroll S, Ingle L. Variability of automated carotid intima-media thickness measurements by novice operators. Clin Physiol Funct Imaging 2014 Sep 12. doi: 10.1111/cpf.12189.
30. Aldridge ME, Do K, Oo T, Naqvi TZ. Carotid intima-media thickness and plaque assessment by trained medical residents: validation and preliminary testing of a training protocol. J Am Soc Echocardiogr 2013; 26: 1457-1464.

31. Vanoli D, Wiklund U, Lindqvist P, Henein M, Näslund U. Successful novice's training in obtaining accurate assessment of carotid IMT using an automated ultrasound system. Eur Heart J Cardiovasc Imaging 2014; 15: 637-642.

32. Kohler TR, Langlois Y, Roederer GO, et al. Variability in measurement of specific parameters for carotid duplex examination. Ultrasound Med Biol 1987; 13: 637-642.

33. Hopkins WG. Measures of reliability in sports medicine and science. Sports Med 2000; 30: 1-15.

34. Atkinson G, Nevill AM. Selected issues in the design and analysis of sport performance research. J Sports Sci 2001; 19: 811-827.

35. Gwet, K. Handbook of Inter-Rater Reliability: The Definitive Guide to Measuring the Extent of Agreement Among Multiple Raters. 3rd Edition. Maryland, USA: Advanced Analytics, LLC, 2012.

36. Lau KH, Fung YK, Cheung YT, Tsang WK, Ying M. Repeatability and reproducibility of ultrasonographic measurement of carotid intima thickness. J Clin Ultrasound 2012; 40: 79-84.

37. Molinari F, Zeng G, Suri JS. A state of the art review on intima-media thickness (IMT) measurement and wall segmentation techniques for carotid ultrasound. Comput Methods Programs Biomed 2010; 100: 201-221.

38. Napel S, Xu H, Paik DS, et al. Carotid disease: automated analysis with cardiac-gated three-dimensional US technique and preliminary results. Radiology 2002; 222: 560-563.

39. Loizou CP, Pattichis CS, Nicolaides AN, Pantziaris M. Manual and automated media and intima thickness measurements of the common carotid artery. IEEE Trans Ultrason Ferroelectr Freq Control 2009; 56: 983-994. 